\title{
Anti-neuron antibody syndrome: clinical features, cytokines/chemokines and predictors
}

Shaohua Liao ${ }^{1+}$, Chuanfen $\mathrm{Li}^{2+}$, Xiaoying Bi ${ }^{1}$, Hongwei Guo ${ }^{3}$, Ying Qian ${ }^{3}$, Xiaobei Liu', Shuai Miao ${ }^{4}$, Huaiqiang $\mathrm{Hu}^{3+}$ and Bingzhen $\mathrm{CaO}^{3{ }^{*+}}$ (1)

\begin{abstract}
Background: Neuroimmunology is a rapidly expanding field, and there have been recent discoveries of new antibodies and neurological syndromes. Most of the current clinical studies have focused on disorders involving one specific antibody. We have summarized a class of antibodies that target common neuronal epitopes, and we have proposed the term "anti-neuron antibody syndrome" (ANAS). In this study, we aimed to clarify the clinical range and analyse the clinical features, cytokines/chemokines and predictors in ANAS.

Methods: This was a retrospective cohort study investigating patients with neurological manifestations that were positive for anti-neuron antibodies.

Results: A total of 110 patients were identified, of which 43 patients were classified as having autoimmune encephalitis (AE) and the other 67 were classified as having paraneoplastic neurological syndrome (PNS). With regards to anti-neuron antibodies, 42 patients tested positive for anti-N-methyl-D-aspartate receptor (NMDAR) antibody, 19 for anti-Hu, 14 for anti-Yo and 12 for anti-PNMA2 (Ma2). There were significant differences between the ANAS and control groups in serum B cell-activating factor (BAFF) levels and in cerebrospinal fluid (CSF) C-X-C motif chemokine10 (CXCL10), CXCL13, interleukin10 (IL10), BAFF and transforming growth factor $\beta 1$ (TGF $\beta 1$ ) levels. Predictors of poor outcomes included having tumours $(P=0.0193)$ and having a chronic onset $(P=0.0306)$, and predictors of relapses included having lower levels of CSF BAFF $(P=0.0491)$ and having a larger ratio of serum TGFB1/serum CXCL13 ( $P=0.0182)$.

Conclusions: Most patients with ANAS had a relatively good prognosis. Having tumours and a chronic onset were both associated with poor outcomes. CSF BAFF and the ratio of serum TGFB1/serum CXCL13 were associated with relapses.
\end{abstract}

Keywords: Anti-neuron antibody syndrome, Autoimmune encephalitis, Paraneoplastic neurological syndrome, Cytokines, Chemokines, Predictors

\footnotetext{
* Correspondence: cbzxia2011@163.com

†Shaohua Liao and Chuanfen Li contributed equally to this work.

${ }^{\dagger}$ Huaiqiang $\mathrm{Hu}$ and Bingzhen Cao contributed equally to this work.

${ }^{3}$ Department of Neurology, 960 Hospital of the PLA Joint Logistics Support Force, Jinan, China

Full list of author information is available at the end of the article
}

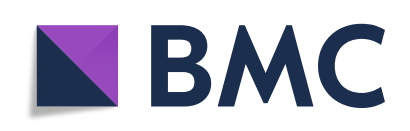

(c) The Author(s). 2021 Open Access This article is licensed under a Creative Commons Attribution 4.0 International License, which permits use, sharing, adaptation, distribution and reproduction in any medium or format, as long as you give appropriate credit to the original author(s) and the source, provide a link to the Creative Commons licence, and indicate if changes were made. The images or other third party material in this article are included in the article's Creative Commons. licence, unless indicated otherwise in a credit line to the material. If material is not included in the article's Creative Commons licence and your intended use is not permitted by statutory regulation or exceeds the permitted use, you will need to obtain permission directly from the copyright holder. To view a copy of this licence, visit http://creativecommons.org/licenses/by/4.0/ The Creative Commons Public Domain Dedication waiver (http://creativecommons.org/publicdomain/zero/1.0/) applies to the data made available in this article, unless otherwise stated in a credit line to the data. 


\section{Introduction}

Neuroimmunology is a rapidly expanding field that has led to the discovery of a series of new antibodies and neurological syndromes. Most of the current clinical studies have focused on disorders related to one specific antibody [1-3] and have limitations regarding the understanding of the generalization of these disorders. We have summarized a class of antibodies and have proposed the term "antineuron antibody syndrome" (ANAS), which is characterized by antibodies that target common neuronal epitopes in certain clinical neurological syndromes. The target antigens mainly include $\mathrm{Hu}, \mathrm{Yo}, \mathrm{NMDAR}$, etc. [4-6] The clinical syndromes consist of autoimmune encephalitis (AE) and paraneoplastic neurological syndromes (PNS).

ANAS is considered to be an immune-mediated disorder and is characterised by the presence of antibodies that target neuronal epitopes. $\mathrm{T}$ and $\mathrm{B}$ cells might be responsible for the syndrome and participate in key steps [4]. Some cytokines/chemokines might play significant roles by affecting the functions of $\mathrm{T}$ and $\mathrm{B}$ cells. Among anti-inflammatory factors, TGF $\beta 1$ and IL10 play important roles by executing their suppressive effects [7, 8]. In proinflammatory elements, CXCL13, CXCL10 and BAFF play crucial roles in the survival, activation and recruitment of $\mathrm{T}$ or B cells [9-11]. The accumulated data suggest that the above factors might affect the development of ANAS by interacting with $\mathrm{T}$ and $\mathrm{B}$ cells.

In this study, we collected the clinical characteristics and data on the cytokines/chemokines in the sera and CSF and aimed to look for the associations between them and ANAS. Then, we strived to identify predictors for the outcomes and relapses of ANAS. Here we report the data.

\section{Methods}

\section{Study design and participants}

We performed a single-centre, retrospective cohort study at the 960 Hospital of the PLA Joint Logistics Support Force. Our study protocol included all patients with neurological manifestations and had positive anti-neuron antibodies, including amphiphysin, CV2, PNMA2, Ri, Yo, $\mathrm{Hu}$, Recoverin, SOX1, Titin, NMDAR and $\mathrm{GABA}_{\mathrm{B}} \mathrm{R}$. Our study included 110 patients hospitalized between March 18, 2011, and July 3, 2017. We collected demographic and clinical data, including age, sex, type of onset, magnetic resonance imaging (MRI) fluid-attenuated inversion recovery (FLAIR)/T2, electroencephalogram (EEG)/electromyogram (EMG), CSF leukocyte counts, CSF protein levels and the antibody-specific diagnosis of the patients. The data with regards to associated tumours, outcomes and relapses were obtained from patients in the hospital and during the follow-up. A total of 108 patients had follow-up evaluations between 24 and 99 months (median 54 months), and 2 patients dropped out. All patients were grouped into AE or PNS by clinical syndromes and into good or poor outcomes by the modified Rankin scale (mRS). They were also grouped into tumours or no tumour and into relapses or no relapse. Good outcomes were defined as a grade of $0-2$ on the mRS, while poor outcomes were defined as a 3-6.

The study was approved by the ethics committee of the 960 Hospital (NO.201611). All patients provided informed written consent to allow their medical records and samples of sera and CSF to be used in this study. All patients' data were processed anonymously.

\section{Samples, antibodies detection and multiplex cytokines/ chemokines immunoassay}

A total of 156 samples were obtained from patients within the first 3 days after admission, which included 105 sera and 51 samples of CSF. The control group included 21 sera that were donated from healthy adults and 20 samples of CSF from apparently healthy adults treated for traumatic fractures of the lower limbs, and the CSF control samples were collected from spinal anaesthesia. All samples were stored at $-80^{\circ} \mathrm{C}$ until analysis.

Testing for anti-NMDAR antibody and anti-GABA ${ }_{B} R$ antibody was performed by recombinant immunofluorescence using an IIFT: Glutamate Receptor Mosaic (Euroimmun, Lubeck, Germany). Paraneoplastic antibodies (i.e., anti-amphiphysin, -CV2, -PNMA2 (Ta), -Ri, -Yo, - Hu, -SOX1, -Titin, -Recoverin) were determined in an immunoblot assay using EUROLINE Neuronal Antigens Profile (IgG) (Euroimmun, Lubeck, Germany). CXCL10, CXCL13, IL10, and BAFF were analysed using a premixed multiplex system (USA R\&D Systems, Inc.) human magnetic luminex assays. TGF- $\beta 1$ was tested using ELISA kits (USA R\&D Systems, Inc.) and a human TGF- $\beta 1$ immunoassay。 The testing was performed according to the manufacturer's instructions. As reported [10, 12], sera were diluted in a 1:5 concentration in the provided buffers, and the CSF was assayed without dilution.

\section{Statistical analysis}

Demographic information and clinical features are expressed as numbers $(\mathrm{N})$ and percentages (\%). Levels of cytokines/chemokines are presented as the median (min, max). The Fisher's exact or Wilcoxon rank sum tests were used to test for significant differences among the different patient groups as appropriate. Variables associated with outcomes or relapses $(p<0.1)$ were included in the following multiple logistic regression analyses. We used a stepwise approach for variable evaluation (backward selection based on likelihood ratio) for the identification of relevant independent variables to be used in the regression model, with a $p$ value of less than 0.05 indicating statistical significance. To facilitate the comparison of effect sizes between cytokines, cytokine distributions were standardized to a mean of 0 and a standard deviation [SD] of 1 . We 
performed all the statistical analyses with SAS, version 9.4 (SAS Institute Inc., Cary NC, USA).

\section{Results}

\section{Patients and clinical features}

A total of 110 patients who tested positive for anti-neuron antibodies were identified in this study. The patients had a median age of 47 years (range: $0.8-78$ years), and 54
(49.1\%) of the patients were women. Fifty-two patients (47.3\%) had an acute onset, 45 (40.9\%) had a chronic onset and $13(11.8 \%)$ had a subacute onset. Thirty-three patients (30\%) were identified as having MRI (FLAIR)/T2 abnormalities (excluding ischaemic lesions), 52 (47.3\%) had EEG/EMG abnormalities, 41 (37.3\%) had a leucocytosis in the CSF (white blood cells $>5 \times 10^{6} / \mathrm{L}$ ) and $30(27.3 \%)$ had elevated CSF protein levels (>450 mg/L) (Table 1).

Table 1 Patient demographics and clinical features, antibodies, tumours in different groups

\begin{tabular}{|c|c|c|c|c|c|c|c|c|c|c|c|c|c|c|c|c|c|c|c|}
\hline \multirow{2}{*}{ Total } & & \multicolumn{2}{|c|}{ Total } & \multicolumn{2}{|c|}{$\mathrm{AE}$} & \multicolumn{2}{|c|}{ PNS } & \multicolumn{2}{|c|}{$\begin{array}{l}\text { No } \\
\text { tumour }\end{array}$} & \multicolumn{2}{|c|}{ Tumours } & \multicolumn{2}{|c|}{$\begin{array}{l}\text { Good } \\
\text { outcomes }\end{array}$} & \multicolumn{2}{|c|}{$\begin{array}{l}\text { Poor } \\
\text { outcomes }\end{array}$} & \multicolumn{2}{|c|}{$\begin{array}{l}\text { No } \\
\text { relapse }\end{array}$} & \multicolumn{2}{|c|}{ Relapses } \\
\hline & & 110 & \#\#\#\#\#\# & 43 & \#\#\#\#\#\# & 67 & \#\#\#\#\#\# & 70 & \#\#\#\#\#\# & 40 & \#\#\#\#\#\# & 73 & (66.36\%) & 37 & (33.64\%) & 77 & \#\#\#\#\#\# & 33 & \#\#\#\#\#\# \\
\hline \multirow[t]{2}{*}{ Age } & $<18$ years & 26 & \#\#\#\#\#\# & 18 & \#\#\#\#\#\# & 8 & (7.27\%) & 24 & \#\#\#\#\#\# & 2 & (1.82\%) & 25 & (22.73\%) & 1 & (0.91\%) & 15 & \#\#\#\#\#\# & 11 & \#\#\#\#\#\# \\
\hline & $\geq 18$ years & 84 & \#\#\#\#\#\# & 25 & \#\#\#\#\#\# & 59 & \#\#\#\#\#\# & 46 & \#\#\#\#\#\# & 38 & \#\#\#\#\#\# & 48 & (43.64\%) & 36 & (32.73\%) & 62 & \#\#\#\#\#\# & 22 & \#\#\#\#\#\# \\
\hline \multirow[t]{2}{*}{ Sex } & Men & 56 & \#\#\#\#\#\# & 19 & \#\#\#\#\#\# & 37 & \#\#\#\#\#\# & 37 & \#\#\#\#\#\# & 19 & \#\#\#\#\#\# & 36 & (32.73\%) & 20 & (18.18\%) & 40 & \#\#\#\#\#\# & 16 & \#\#\#\#\#\# \\
\hline & Women & 54 & \#\#\#\#\#\# & 24 & \#\#\#\#\#\# & 30 & \#\#\#\#\#\# & 33 & \#\#\#\#\#\# & 21 & \#\#\#\#\#\# & 37 & (33.64\%) & 17 & (15.45\%) & 37 & \#\#\#\#\#\# & 17 & \#\#\#\#\#\# \\
\hline \multirow{3}{*}{$\begin{array}{l}\text { Types of } \\
\text { onset }\end{array}$} & Acute & 52 & \#\#\#\#\#\# & 34 & \#\#\#\#\#\# & 18 & \#\#\#\#\#\# & 36 & \#\#\#\#\#\# & 16 & \#\#\#\#\#\# & 43 & (39.09\%) & 9 & $(8.18 \%)$ & 31 & \#\#\#\#\#\# & 21 & \#\#\#\#\#\# \\
\hline & Sub-acute & 13 & \#\#\#\#\#\# & 4 & (3.64\%) & 9 & (8.18\%) & 10 & (9.09\%) & 3 & (2.73\%) & 9 & (8.18\%) & 4 & (3.64\%) & 9 & (8.18\%) & 4 & (3.64\%) \\
\hline & Chronic & 45 & \#\#\#\#\#\# & 5 & $(4.55 \%)$ & 40 & \#\#\#\#\#\# & 24 & \#\#\#\#\#\# & 21 & \#\#\#\#\#\# & 21 & (19.09\%) & 24 & (21.82\%) & 37 & \#\#\#\#\#\# & 8 & (7.27\%) \\
\hline \multirow{2}{*}{$\begin{array}{l}\text { MRI Flair/T2 } \\
\text { abnormality }\end{array}$} & No & 66 & \#\#\#\#\#\# & 29 & \#\#\#\#\#\# & 37 & \#\#\#\#\#\# & 41 & \#\#\#\#\# & 25 & \#\#\#\#\#\# & 42 & (38.18\%) & 24 & (21.82\%) & 52 & \#\#\#\#\#\# & 14 & \#\#\#\#\#\# \\
\hline & Yes & 33 & \#\#\#\#\#\# & 12 & \#\#\#\#\#\# & 21 & \#\#\#\#\#\# & 23 & \#\#\#\#\#\# & 10 & (9.09\%) & 23 & (20.91\%) & 10 & (9.09\%) & 19 & \#\#\#\#\#\# & 14 & \#\#\#\#\#\# \\
\hline \multirow{2}{*}{$\begin{array}{l}\text { EEG/EMG } \\
\text { abnormality }\end{array}$} & No & 7 & $(6.36 \%)$ & 5 & $(4.55 \%)$ & 2 & $(1.82 \%)$ & 5 & $(4.55 \%)$ & 2 & $(1.82 \%)$ & 6 & $(5.45 \%)$ & 1 & $(0.91 \%)$ & 3 & $(2.73 \%)$ & 4 & $(3.64 \%)$ \\
\hline & Yes & 52 & \#\#\#\#\#\# & 22 & \#\#\#\#\#\# & 30 & \#\#\#\#\#\# & 36 & \#\#\#\#\#\# & 16 & \#\#\#\#\#\# & 34 & (30.91\%) & 18 & (16.36\%) & 40 & \#\#\#\#\#\# & 12 & \#\#\#\#\#\# \\
\hline \multirow{2}{*}{$\begin{array}{l}\text { CSF } \\
\text { leukocytosis }\end{array}$} & No & 42 & \#\#\#\#\#\# & 15 & \#\#\#\#\#\# & 27 & \#\#\#\#\#\# & 28 & \#\#\#\#\#\# & 14 & \#\#\#\#\#\# & 33 & (30.00\%) & 9 & (8.18\%) & 28 & \#\#\#\#\#\# & 14 & \#\#\#\#\#\# \\
\hline & Yes & 41 & \#\#\#\#\#\# & 23 & \#\#\#\#\#\# & 18 & \#\#\#\#\#\# & 28 & \#\#\#\#\#\# & 13 & \#\#\#\#\#\# & 24 & (21.82\%) & 17 & (15.45\%) & 28 & \#\#\#\#\#\# & 13 & \#\#\#\#\#\# \\
\hline \multirow{2}{*}{$\begin{array}{l}\text { Elevated } \\
\text { CSF PRO }\end{array}$} & No & 53 & \#\#\#\#\#\# & 30 & \#\#\#\#\#\# & 23 & \#\#\#\#\#\# & 38 & \#\#\#\#\#\# & 15 & \#\#\#\#\#\# & 42 & (38.18\%) & 11 & (10.00\%) & 29 & \#\#\#\#\#\# & 24 & \#\#\#\#\#\# \\
\hline & Yes & 30 & \#\#\#\#\#\# & 8 & $(7.27 \%)$ & 22 & \#\#\#\#\#\# & 18 & \#\#\#\#\#\# & 12 & \#\#\#\#\#\# & 15 & (13.64\%) & 15 & (13.64\%) & 27 & \#\#\#\#\#\# & 3 & (2.73\%) \\
\hline \multirow{2}{*}{$\begin{array}{l}\text { CSF } \\
\text { abnormality }\end{array}$} & No & 31 & \#\#\#\#\#\# & 14 & \#\#\#\#\#\# & 17 & \#\#\#\#\#\# & 22 & \#\#\#\#\#\# & 9 & (8.18\%) & 25 & (22.73\%) & 6 & (5.45\%) & 18 & \#\#\#\#\#\# & 13 & \#\#\#\#\#\# \\
\hline & Yes & 52 & \#\#\#\#\#\# & 24 & \#\#\#\#\#\# & 28 & \#\#\#\#\#\# & 34 & \#\#\#\#\#\# & 18 & \#\#\#\#\#\# & 32 & (29.09\%) & 20 & (18.18\%) & 38 & \#\#\#\#\#\# & 14 & \#\#\#\#\#\# \\
\hline \multirow{8}{*}{$\begin{array}{l}\text { Anti-neuron } \\
\text { antibodies }\end{array}$} & Anti-NMDAR & 42 & \#\#\#\#\#\# & 42 & \#\#\#\#\#\# & 0 & $(0.00 \%)$ & 35 & \#\#\#\#\#\# & 7 & $(6.36 \%)$ & 36 & $(32.73 \%)$ & 6 & $(5.45 \%)$ & 23 & \#\#\#\#\#\# & 19 & \#\#\#\#\#\# \\
\hline & Anti-Hu & 19 & \#\#\#\#\#\# & 0 & $(0.00 \%)$ & 19 & \#\#\#\#\#\# & 7 & $(6.36 \%)$ & 12 & \#\#\#\#\#\# & 6 & $(5.45 \%)$ & 13 & (11.82\%) & 14 & \#\#\#\#\#\# & 2 & (1.82\%) \\
\hline & Anti-Yo & 14 & \#\#\#\#\#\# & 0 & $(0.00 \%)$ & 14 & \#\#\#\#\#\# & 9 & $(8.18 \%)$ & 5 & $(4.55 \%)$ & 6 & $(5.45 \%)$ & 8 & (7.27\%) & 10 & $(9.09 \%)$ & 4 & $(3.64 \%)$ \\
\hline & Anti-PNMA2 & 12 & \#\#\#\#\#\# & 0 & $(0.00 \%)$ & 12 & \#\#\#\#\#\# & 8 & $(7.27 \%)$ & 4 & (3.64\%) & 10 & $(9.09 \%)$ & 2 & $(1.82 \%)$ & 10 & (9.09\%) & 2 & (1.82\%) \\
\hline & Anti-CV2 & 8 & (7.27\%) & 0 & $(0.00 \%)$ & 8 & $(7.27 \%)$ & 2 & $(1.82 \%)$ & 6 & $(5.45 \%)$ & 4 & (3.64\%) & 4 & (3.64\%) & 7 & (6.36\%) & 1 & (0.91\%) \\
\hline & $\begin{array}{l}\text { Anti- } \\
\text { Amphiphysin }\end{array}$ & 8 & $(7.27 \%)$ & 0 & $(0.00 \%)$ & 8 & $(7.27 \%)$ & 2 & $(1.82 \%)$ & 6 & $(5.45 \%)$ & 4 & $(3.64 \%)$ & 4 & $(3.64 \%)$ & 7 & $(6.36 \%)$ & 1 & (0.91\%) \\
\hline & Anti-SOX1 & 7 & $(6.36 \%)$ & 0 & $(0.00 \%)$ & 7 & $(6.36 \%)$ & 4 & (3.64\%) & 3 & $(2.73 \%)$ & 3 & $(2.73 \%)$ & 4 & (3.64\%) & 5 & $(4.55 \%)$ & 2 & (1.82\%) \\
\hline & Others & 7 & $(6.36 \%)$ & 1 & $(0.91 \%)$ & 6 & $(5.45 \%)$ & 4 & $(3.64 \%)$ & 3 & $(2.73 \%)$ & 7 & $(6.36 \%)$ & 0 & $(0.00 \%)$ & 4 & $(3.64 \%)$ & 3 & $(2.73 \%)$ \\
\hline \multirow[t]{5}{*}{ Tumours } & $\begin{array}{l}\text { Non-small } \\
\text { cell lung } \\
\text { cancer }\end{array}$ & 9 & $(8.18 \%)$ & 0 & $(0.00 \%)$ & 9 & (8.18\%) & 0 & $(0.00 \%)$ & 9 & $(8.18 \%)$ & 1 & (0.91\%) & 8 & $(7.27 \%)$ & 9 & $(8.18 \%)$ & 0 & $(0.00 \%)$ \\
\hline & $\begin{array}{l}\text { Small cell } \\
\text { lung cancer }\end{array}$ & 6 & $(5.45 \%)$ & 1 & $(0.91 \%)$ & 5 & $(4.55 \%)$ & 0 & $(0.00 \%)$ & 6 & (5.45\%) & 2 & $(1.82 \%)$ & 4 & (3.64\%) & 5 & $(4.55 \%)$ & 1 & (0.91\%) \\
\hline & Breast cancer & 5 & $(4.55 \%)$ & 0 & $(0.00 \%)$ & 5 & $(4.55 \%)$ & 0 & $(0.00 \%)$ & 5 & $(4.55 \%)$ & 1 & (0.91\%) & 4 & (3.64\%) & 3 & $(2.73 \%)$ & 2 & $(1.82 \%)$ \\
\hline & $\begin{array}{l}\text { Ovarian } \\
\text { teratoma }\end{array}$ & 3 & (2.73\%) & 3 & $(2.73 \%)$ & 0 & $(0.00 \%)$ & 0 & $(0.00 \%)$ & 3 & $(2.73 \%)$ & 3 & $(2.73 \%)$ & 0 & $(0.00 \%)$ & 1 & $(0.91 \%)$ & 2 & $(1.82 \%)$ \\
\hline & Others & 18 & \#\#\#\#\#\# & 3 & $(2.73 \%)$ & 15 & \#\#\#\#\#\# & 0 & $(0.00 \%)$ & 15 & \#\#\#\#\#\# & 9 & (8.18\%) & 9 & (8.18\%) & 13 & \#\#\#\#\#\# & 5 & $(4.55 \%)$ \\
\hline
\end{tabular}

Data are $\mathrm{n}(\%)$

$A E$ autoimmune encephalitis, PNS paraneoplastic neurological syndromes, MRI FLAIR Magnetic resonance imaging fluid-attenuated inversion recovery, EEG electroencephalogram, EMG electromyogram, CSF cerebrospinal fluid, PRO protein, NMDAR N-methyl-D-aspartate receptor 

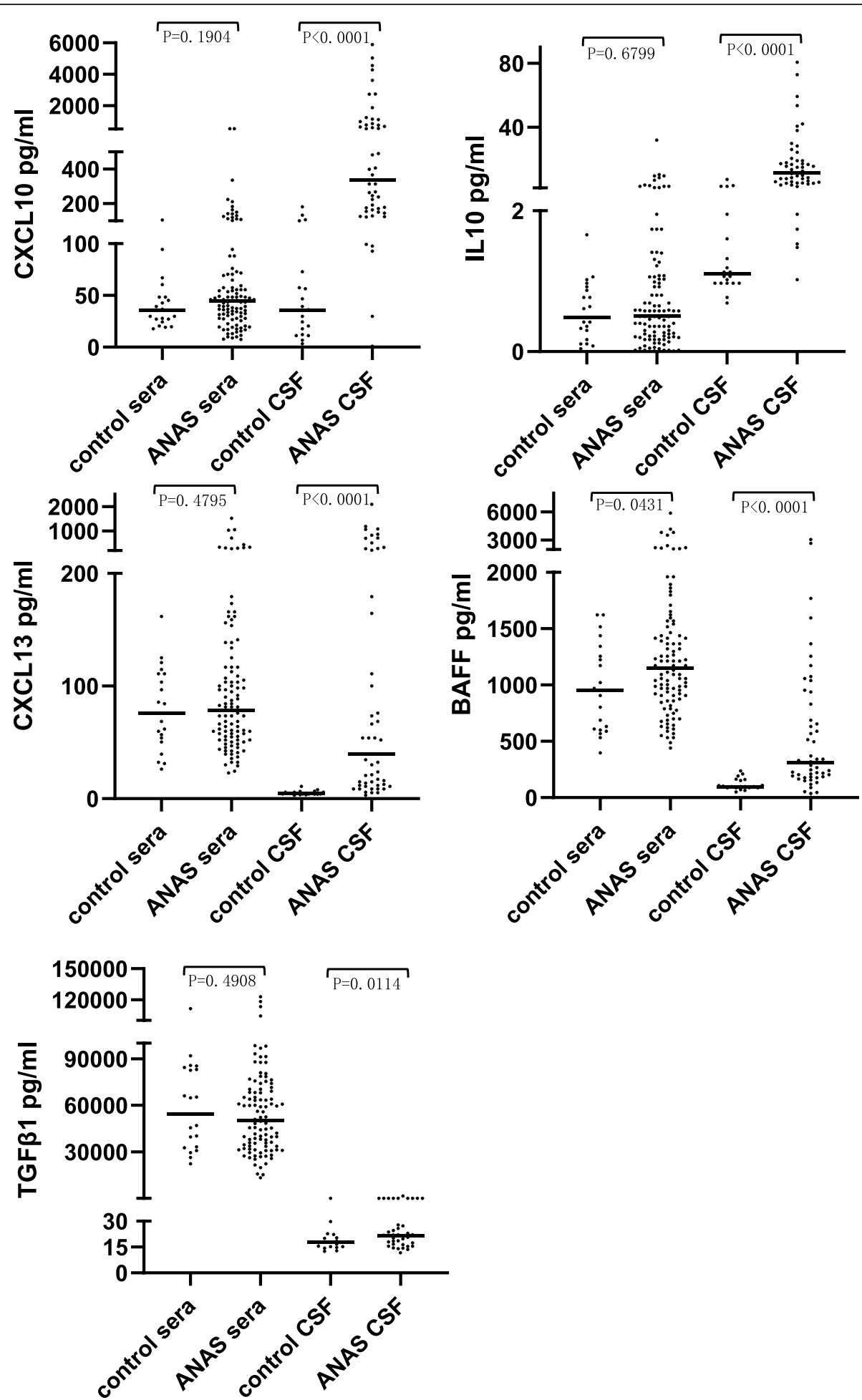

Fig. 1 Cytokines/chemokines levels in sera and CSF compared between the ANAS and control group. There were significant differences in serum BAFF levels $(P=0.0431)$ and in CSF CXCL10, CXCL13, IL10, BAFF and TGF $\beta 1$ levels $(P<0.05)$ between the ANAS and control group

Regarding the anti-neuron antibodies in the patients, 42 patients $(38.2 \%)$ tested positive for anti-NMDAR antibodies, 19(17.3\%)for anti-Hu, 14 (12.7\%)for anti-Yo,
$12(10.9 \%)$ for anti-PNMA2, 8(7.3\%) for antiAmphiphysin, 8 (7.3\%) for anti-CV2 and 7 for others (6.4\%). As reported [13], 43 (39.1\%) patients (42 for anti- 
NMDAR and 1 for anti-GABA $\mathrm{R}$ ) were classified as $\mathrm{AE}$, and the other 67 patients $(60.9 \%)$ were classified as PNS. Factors significantly associated with PNS patients included having a chronic onset $(P<0.0001)$, an elevated CSF protein level $(P=0.0116)$, tumours $(P=0.0005)$, no relapse $(P=0.0113)$ and poor outcomes $(P=0.0004)$ when compared to $\mathrm{AE}$ patients. In this study, 40(36.4\%) patients had a tumour. Nine tumours $(8.2 \%)$ were nonsmall cell lung cancers, 6 (5.5\%) were small cell lung cancers, 5 (4.5\%) were breast cancers, 3 (2.7\%) were ovarian teratomas and 17 (15.5\%) were other tumours.
At the final follow-up, 37 patients (33.6\%) had poor outcomes. In patients with the poor outcomes, we recorded significant relevant factors, including having a chronic onset $(P=0.0002)$, an elevated CSF protein level $(P=0.0075)$, tumours $(P<0.0001)$ and no relapse $(P=0.0081)$. During the follow-up, 33 patients (30\%) had clinical relapses, including 23 patients $(23 / 30,76.7 \%)$ that relapsed once and 1 patient that relapsed 8 times. We also recorded significant positive correlations between having a relapse and an acute onset $(P=0.0163)$, MRI (FLAIR)/T2 abnormalities $(P=0.0345)$ and a normal CSF protein level $(P=0.0012)$.
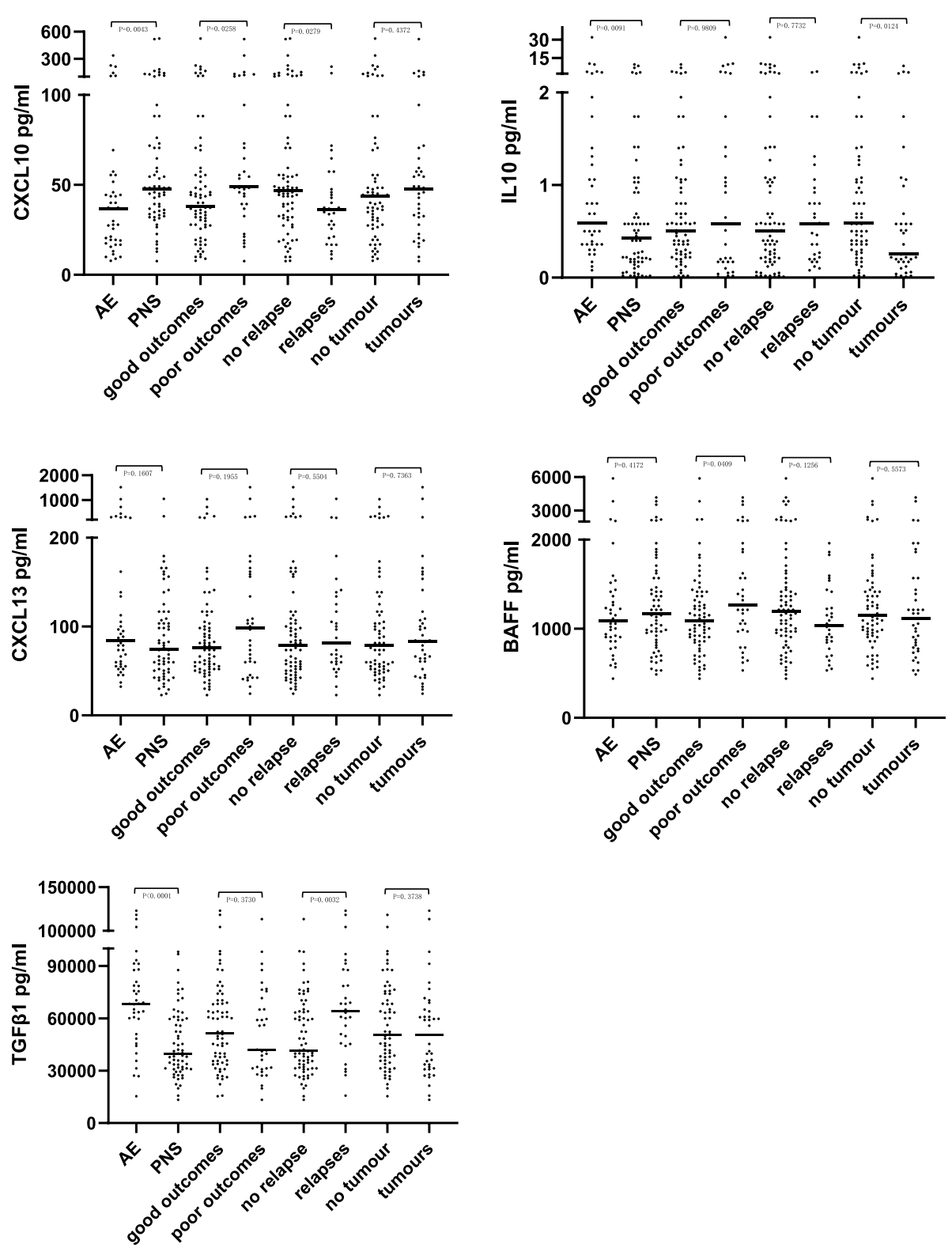

Fig. 2 Cytokines/chemokines levels in sera compared within different ANAS groups. In AE compared to PNS, there were significant differences in CXCL10 $(P=0.0043), I L 10(P=0.0091)$ and TGF $\beta 1(P<0.0001)$. There were significant inverse relationships between IL10 levels and tumours $(P=$ 0.0124). And significant positive correlations between $C X C L 10(P=0.0258)$, BAFF $(P=0.0409)$ levels and poor outcomes. And significant positive correlations for TGF $\beta 1$ levels $(P=0.0032)$, significant inverse relationships for CXCL10 levels $(P=0.0279)$ in patients with relapses 


\section{Analysis of cytokines/chemokines}

In this study, we collected 105 serum samples and 51 samples of CSF from ANAS patients and 21 serum samples and 20 samples of CSF from the control group. There were significant differences in serum BAFF levels $(P=0.0431)$ and in CSF CXCL10, CXCL13, IL10, BAFF and TGF $\beta 1$ levels $(P<0.05)$ between the ANAS and control group (Fig. 1).

In the univariate analysis of the serum cytokines/chemokines levels, there were significant differences in CXCL10 $(P=0.0043), \operatorname{IL10}(P=0.0091)$ and TGF $\beta 1(P$ $<0.0001)$ between the AE and PNS patients. We detected significant inverse relationships between IL10 levels and patients with tumours $(P=0.0124)$. In patients with poor outcomes, the significant relevant factors included higher levels of CXCL10 $(P=0.0258)$ and BAFF $(P=0.0409)$ than those with good outcomes. In patients with relapses, we recorded significant positive correlations for TGF $\beta 1(P=0.0032)$, whereas we detected significant inverse relationships for CXCL10 $(P=$ 0.0279) compared with no relapse (Fig. 2). For the CSF cytokines/chemokines levels, there were no significant differences between the different groups $(P>0.05)$.

\section{Predictors of outcomes and relapses}

In all patients, the univariate analysis revealed factors associated with poor outcomes, including having a chronic onset $(P=0.0002)$, an elevated CSF protein level $(P=$ $0.0075)$, tumours $(P<0.0001)$, no relapse $(P=0.0081)$, PNS $(P=0.0004)$, higher levels of serum CXCL10 $(P=$ $0.0258)$ and serum BAFF $(P=0.0409)$ (Table 2). Then, the logistic multivariate stepwise regression analysis indicated the factors that were associated with poor outcomes, and these included having tumours (OR 10.156, CI 1.458-70.748, $P=0.0193$ ) and a chronic onset (OR 13.921, CI 1.279-151.586, $P=0.0306$ ) (Table 4). As mentioned above, in the univariate analysis, factors associated with relapses included having a chronic onset $(P$ $=0.0163)$, MRI (FLAIR) $/$ T2 abnormalities $(P=0.0345)$, a normal CSF protein level $(P=0.0012), \operatorname{AE}(P=$ $0.0113)$, a good outcome $(P=0.0081)$, lower levels of serum CXCL10 $(P=0.0279)$ and higher levels of serum

Table 2 In 110 ANAS patients, factors associated with outcomes or relapses in univariate analysis

\begin{tabular}{|c|c|c|c|c|c|c|c|}
\hline \multicolumn{4}{|l|}{ Outcomes } & \multicolumn{4}{|l|}{ Relapses } \\
\hline & Good outcomes & Poor outcomes & $p$ & & NO relapose & Relapses & $p$ \\
\hline Types of onset & & & 0.0002 & Types of onset & & & 0.0163 \\
\hline Acute & $43(58.90 \%)$ & $9(24.32 \%)$ & & Acute & $31(40.26 \%)$ & $21(63.64 \%)$ & \\
\hline Sub-acute & $9(12.33 \%)$ & 4(10.81\%) & & Sub-acute & $9(11.69 \%)$ & $4(12.12 \%)$ & \\
\hline Chronic & $21(28.77 \%)$ & $24(64.86 \%)$ & & Chronic & $37(48.05 \%)$ & $8(24.24 \%)$ & \\
\hline Elevated CSF PRO & & & 0.0075 & Elevated CSF PRO & & & 0.0012 \\
\hline No & $42(73.68 \%)$ & $11(42.31 \%)$ & & NO & 29(51.79\%) & 24(88.89\%) & \\
\hline Yes & $15(26.32 \%)$ & $15(57.69 \%)$ & & YES & $27(48.21 \%)$ & $3(11.11 \%)$ & \\
\hline Tumours & & & $<0.0001$ & $\begin{array}{l}\text { MRI Flair/T2 } \\
\text { abnormalities }\end{array}$ & & & 0.0345 \\
\hline No & $57(81.43 \%)$ & 13(18.57\%) & & NO & $52(73.24 \%)$ & $14(50.00 \%)$ & \\
\hline Yes & $16(40.00 \%)$ & $24(60.00 \%)$ & & YES & $19(26.76 \%)$ & $14(50.00 \%)$ & \\
\hline AE or PNS & & & 0.0004 & AE or PNS & & & 0.0113 \\
\hline$A E$ & $37(50.68 \%)$ & $6(16.22 \%)$ & & $\mathrm{AE}$ & 24(55.81\%) & 19(44.19\%) & \\
\hline PNS & $36(49.32 \%)$ & $31(83.78 \%)$ & & PNS & $53(79.10 \%)$ & $14(20.90 \%)$ & \\
\hline Serum CXCL10 & $\begin{array}{l}38.02(7.84- \\
525.63)\end{array}$ & $\begin{array}{l}48.77(7.58- \\
516.68)\end{array}$ & 0.0258 & Serum CXCL10 & $\begin{array}{l}46.73(7.58- \\
525.63)\end{array}$ & $\begin{array}{l}36.11(9.00- \\
211.62)\end{array}$ & 0.0279 \\
\hline Serum BAFF & $\begin{array}{l}1094(440.36- \\
5882.00)\end{array}$ & $\begin{array}{l}1266.5(531.88- \\
4173.00)\end{array}$ & 0.0409 & Serum TGF $\beta 1$ & $\begin{array}{l}41401.57(13345.38- \\
113337.9)\end{array}$ & $\begin{array}{l}\text { 64140.37(15755.87- } \\
122994.8)\end{array}$ & 0.0032 \\
\hline CSF leukocytosis & & & 0.0606 & & & & \\
\hline No & 33(57.89\%) & $9(34.62 \%)$ & & & & & \\
\hline Yes & $24(42.11 \%)$ & $17(65.38 \%)$ & & & & & \\
\hline CSF abnormality & & & 0.0889 & & & & \\
\hline No & $25(43.86 \%)$ & $6(23.08 \%)$ & & & & & \\
\hline Yes & $32(56.14 \%)$ & 20(76.92\%) & & & & & \\
\hline
\end{tabular}

Counting data are $\mathrm{n}(\%)$, and measruement data are median (min, max)

CSF cerebrospinal fluid, PRO protein, $A E$ autoimmune encephalitis, PNS paraneoplastic neurological syndromes, $C X C L C-X-C$ motif chemokine, $B A F F$ B cell activating factor, MRI FLAIR Magnetic resonance imaging fluid-attenuated inversion recovery, TGF $\beta 1$ transforming growth factor $\beta 1$, ANAS anti-neuron antibody syndrome 
Table 3 In 51 ANAS patients with CSF, factors associated with outcomes or relapses in univariate analysis

\begin{tabular}{|c|c|c|c|c|c|c|c|}
\hline \multicolumn{4}{|l|}{ Outcomes } & \multicolumn{4}{|l|}{ Relapses } \\
\hline & $\begin{array}{l}\text { Good } \\
\text { outcomes }\end{array}$ & $\begin{array}{l}\text { Poor } \\
\text { outcomes }\end{array}$ & $p$ & & No relapse & Relapses & $p$ \\
\hline $\begin{array}{l}\text { Elevated CSF } \\
\text { PRO }\end{array}$ & & & 0.0123 & Elevated CSF PRO & & & 0.0632 \\
\hline No & $29(76.32)$ & 4(33.33) & & No & $17(54.84)$ & $16(84.21)$ & \\
\hline Yes & $9(23.68)$ & $8(66.67)$ & & Yes & 14(45.16) & $3(15.79)$ & \\
\hline AE or PNS & & & 0.0372 & AE or PNS & & & 0.0737 \\
\hline$A E$ & 28(71.79) & 4(33.33) & & AE & $16(51.61)$ & 16(80.00) & \\
\hline PNS & $11(28.21)$ & $8(66.67)$ & & PNS & 15(48.39) & $4(20.00)$ & \\
\hline Tumours & & & $<0.0001$ & CSF IL10 & $14.99(1.48-80.63)$ & 7.8(1.02-28.29) & 0.0913 \\
\hline No & $36(92.31)$ & 4(33.33) & & & & & \\
\hline Yes & $3(7.69)$ & $8(66.67)$ & & & & & \\
\hline Types of onset & & & 0.001 & Serum TGF $\beta 1$ & $\begin{array}{l}52379.98(15370.86- \\
113337.9)\end{array}$ & $\begin{array}{l}\text { 69013.08(27451.21- } \\
118364.7)\end{array}$ & 0.0202 \\
\hline Acute & $32(82.05)$ & $4(33.33)$ & & & & & \\
\hline Sub-acute & $2(5.13)$ & $1(8.33)$ & & & & & \\
\hline Chronic & $5(12.82)$ & $7(58.33)$ & & & & & \\
\hline \multirow[t]{6}{*}{ CSF BAFF } & $\begin{array}{l}267.68 \\
(2.54-3064.00)\end{array}$ & $\begin{array}{l}742.31 \\
(89.23-2670.00)\end{array}$ & 0.0606 & CSF BAFF & $370.9(2.54-3064.00)$ & 242.77(36.56-1367.00) & 0.099 \\
\hline & & & & $\begin{array}{l}\text { Serum TGF } \beta 1 / \text { CSF } \\
\text { CXCL10 }\end{array}$ & 102.48(7.33-927.55) & $310.13(6.02-3235.45)$ & 0.0048 \\
\hline & & & & $\begin{array}{l}\text { Serum TGF } \beta 1 / \text { serum } \\
\text { CXCL13 }\end{array}$ & 574.07(70.48,2913.36) & $1041.71(264.89,1618.54)$ & 0.0455 \\
\hline & & & & $\begin{array}{l}\text { Serum TGF } \beta 1 / \text { serum } \\
\text { BAFF }\end{array}$ & 41.76(5.34-113.62) & $64.24(21.85-140.59)$ & 0.0279 \\
\hline & & & & CSF TGF $\beta 1 /$ serum TGF $\beta 1$ & $0.00064(0.00012-0.049)$ & $0.00025(0.00014-0.0016)$ & 0.0205 \\
\hline & & & & Serum TGF $\beta 1 / C S F$ BAFF & $114.29(16.12-1043.37)$ & $359.88(55.28-2646.06)$ & 0.0076 \\
\hline
\end{tabular}

Counting data are $\mathrm{n}(\%)$, and measruement data are median ( $\min , \max )$

CSF cerebrospinal fluid, $P R O$ protein, AE autoimmune encephalitis, PNS paraneoplastic neurological syndromes, BAFF B cell activating factor, IL interleukin, TGF $\beta 1$ transforming growth factor $\beta 1, C X C L$ C-X-C motif chemokine, ANAS anti-neuron antibody syndrome

TGFß1 $(P=0.0032)$ (Table 2). In the multivariate analysis, none of the factors was associated with relapses $(P$ $>0.05)$.

In the 51 patients with CSF cytokines/chemokines data, the ratios of two cytokine/chemokine levels were possible factors that might be associated with outcomes or relapses. In the univariate analysis, factors associated with poor outcomes included having higher levels of
CSF BAFF $(P=0.0606)$, a chronic onset $(P=0.0010)$, an elevated CSF PRO level $(P=0.0123)$, tumours $(P<$ $0.0001)$, and PNS $(P=0.0372)$ (Table 3$)$. In the multivariate analysis, the factor associated with poor outcomes was tumours (OR 13.859, CI 2.242-85.683, $P=$ 0.0047 ) (Table 4). In the univariate analysis, factors associated with relapses included larger ratios of serum TGF $\beta 1 /$ CSF CXCL10 $(P=0.0048)$, serum TGF $\beta 1 /$ serum

Table 4 Predictors of outcomes or relapses in ANAS patients in multivariate analysis

\begin{tabular}{|c|c|c|c|c|c|}
\hline \multicolumn{3}{|c|}{ In 110 ANAS patients } & \multicolumn{3}{|l|}{ In 51 ANAS patients with CSF } \\
\hline Outcomes & $\mathrm{OR}(95 \% \mathrm{Cl})$ & $p$ & Outcomes & OR(95\%Cl) & $p$ \\
\hline Tumours & $10.156(1.458-70.748)$ & 0.0193 & Tumours & $13.859(2.242-85.683)$ & 0.0047 \\
\hline \multirow[t]{4}{*}{ Types of onset } & 13.921(1.279-151.586) & 0.0306 & & & \\
\hline & & & Relapses & Per-SD increase [OR(95\%Cl)] & $p$ \\
\hline & & & serum TGF $\beta 1 /$ serum CXCL13 & 5.809 (1.349-25.002) & 0.0182 \\
\hline & & & CSF BAFF & $0.038(0.001-0.988)$ & 0.0491 \\
\hline
\end{tabular}


CXCL13 $(P=0.0455)$, serum TGF $\beta 1 /$ serum BAFF $(P=$ $0.0279)$, CSF TGF $\beta 1 /$ serum TGF $\beta 1(P=0.0205)$, serum TGF $\beta 1 /$ CSF BAFF $(P=0.0076)$, higher levels of serum TGF $\beta 1(P=0.0202)$ and other factors were identified that might be associated with relapses, including having a normal CSF protein level $(P=0.0632), \operatorname{AE}(P=$ 0.0737), lower levels of CSF IL10 $(P=0.0913)$ and CSF BAFF $(P=0.0990)$ (Table 3$)$. In multivariate analysis, the factors associated with relapses included lower levels of CSF BAFF (OR 0.038, CI 0.001-0.988, $P=0.0491$ ) and a larger ratio of serum TGF $\beta 1 /$ serum CXCL13 (OR 5.809, CI 1.349-25.002, $P=0.0182$ ) (Table 4).

\section{Discussion}

Neuroimmunology is a rapidly expanding field in neuroscience that has led to a paradigmatic shift in the understanding of immunological diseases in the CNS and has led to the discovery of a series of new antibodies and neurological syndromes. This study was a retrospective analysis based on the clinical data of our centre in the past 7 years, which included some new insights to antineuron antibodies and related neurological syndrome. ANAS is a clinical neurological syndrome that is characterized by the presence of antibodies that target neuronal epitopes. As reported, the target antigens include nuclear or cytoplasmic proteins, such as $\mathrm{Hu}, \mathrm{Ri}$, Yo, Ma2, ANNA-3, MAP1B, SOX1, CRMP5, ZIC4, NIF, ITPR1 [4, 14], intracellular synaptic proteins, such as 65 $\mathrm{kDa}$ glutamic acid decarboxylase (GAD65) and amphiphysin, and cell-surface or synaptic proteins, such as NMDAR, AMPAR, $\gamma$-aminobutyric acid receptor-B (GABAR), LGI1, Caspr2, GIyR, mGIuR5 [4], DPPX [1], DR2 [2], Iglon5 [3] and neurexin-3 $\alpha$ [15]. The main syndromes are classified as AE and PNS, which is based on the widely recognized methods in recent years $[14,16$, 17]. The term ANAS is not intended to be used as a clinical diagnosis, but the recognition of this syndrome is useful for the understanding of the common aetiologies and pathogeneses. This study aimed to summarize the general characteristics and clarify the clinical range of ANAS, which is beneficial for clinical neuroscientists to understand the aetiological relationships and pathogeneses of diseases associated with ANAS.

The study clarified the clinical range of ANAS, summarized the data, including clinical features, outcomes and relapses, and then focused on the cytokines/chemokines found in the sera and CSF, so as to provide the basis for doctors' clinical decision-making and to help analyse the related factors of the prognosis. In the univariate and logistic multivariate stepwise regression analyses, the study revealed that possible factors that were associated with poor outcomes included tumours and having a chronic onset, and possible factors associated with relapses included having lower levels of CSF BAFF and a larger ratio of serum TGF $\beta 1 /$ serum CXCL13. These factors might be predictors for ANAS.

In these cases, $47.3 \%$ of patients had acute onset, 30\% had MRI FLAIR/T2 abnormalities, 47.3\% had EEG/EMG abnormalities and $27.3 \%$ had elevated CSF protein. In a cohort study of anti-NMDAR encephalitis [18], MRI of the brain and EEG and CSF studies were abnormal in $33 \%, 90 \%$ and $79 \%$ of patients, respectively. The results suggested that these clinical features might not be specific for ANAS, and the diagnosis might require antibody detection.

There were more patients (60.9\%) classified as PNS, and the common antibodies found were anti-NMDAR, anti-Hu and anti-Yo. A total of $36.4 \%$ of patients had tumours, and the majority of the tumours were lung cancers and breast cancers. As shown in other research on PNS, the common antibodies and tumours were anti-Yo, anti- $\mathrm{Hu}$, and anti-titin antibodies and thymoma and lung cancers, respectivel y[19], which were partially consistent with our study. The inconsistency may have something to do with the different populations studied, including differences in the participants' races. There were $66.4 \%$ of patients with good outcomes and 70\% without relapses in our study, which indicated a relatively good prognosis for ANAS.

In general, ANAS is considered to be an immunemediated disorder and has certain antibodies targeting neuronal epitopes. $\mathrm{T}$ and $\mathrm{B}$ cells might be responsible for the syndrome and participate in key steps [4]. Some cytokines/chemokines might play significant roles in the occurrence and development of ANAS by affecting the functions of $\mathrm{T}$ and $\mathrm{B}$ cells. Among anti-inflammatory factors, TGF- $\beta 1$ and IL-10 play important roles in the development of inflammation in the peripheral and central nervous systems. TGF- $\beta 1$ exerts its suppressive effects by inhibiting cytotoxic $\mathrm{T}$ cells, Th1 and Th2 cell differentiation and controlling $\mathrm{B}$ cell proliferation, survival, activation and differentiation [7]. IL-10 plays a pivotal role in restraining the immune response on myeloid cells by suppressing proinflammatory cytokines and antigen-presenting cells (APCs) [8]. In proinflammatory elements, CXCL13, CXCL10 and BAFF play crucial roles in the survival, activation and recruitment of $\mathrm{T}$ or $\mathrm{B}$ cells. CXCL13 is considered to be the major determinant for B cell recruitment to the CNS compartment in neuroinflammatory diseases, especially in the CSF [9]. It is also mildly elevated in multiple sclerosis (MS) and neuromyelitis optica $[20,21]$. CXCL10 recruits $\mathrm{CXCR}^{+}$ cells, such as activated T cells, in CNS inflammation,[10] and they were found to colocalize with active MS lesions [22]. BAFF is a member of the tumour necrosis factor (TNF) family and acts as an amplifier of autoimmune responses by promoting the activation and survival of $\mathrm{B}$ cells [11]. The accumulated data suggest that the above 
factors might interact with $\mathrm{T}$ and $\mathrm{B}$ cells and affect the pathogenesis of ANAS

The measurement of cytokines/chemokines in the sera and CSF were the key points of the study. Our study aimed to screen several cytokines/chemokines as predictors of the outcomes and relapses of ANAS. As reported in previous studies [10, 23], PNS patients had high levels of the chemokine CXCL10 in the CSF. Elevated soluble Fas and FasL have been found in the CSF and sera of patients with anti-NMDAR encephalitis [13]. Certain key cytokines/chemokines might act as biomarkers of inflammation and the response to treatments in the CNS [12, 24]. These studies demonstrated that some cytokines/chemokines may be related to ANAS and influence the prognosis of this syndrome.

In this study, some cytokines/chemokines related to the activation or inhibition of $\mathrm{T}$ and $\mathrm{B}$ cells were observed. We tested the levels of cytokines/chemokines in the CSF and sera and then further investigated the relationship between cytokines/chemokines and ANAS. In the univariate analysis, the elevation of CXCL10 and BAFF in the sera indicated a poor outcome, and a decrease of CXCL10 and an elevation of TGF $\beta 1$ in the sera was associated with relapses. In the multivariate analysis, the factors associated with relapses included lower levels of CSF BAFF and a larger ratio of serum TGF $\beta 1 /$ serum CXCL13. These cytokines/ chemokines might be predictors for outcomes and relapses of ANAS. In this research, we aimed to identify some cytokines/chemokines that were able to predict the prognosis. We also explored more associated factors and calculations that might be related to the prognosis. The ratios of two cytokine/chemokine levels were counted as factors. Then, we observed whether they were associated with outcomes and relapses in the univariate and multivariate analyses. In our study, the ratio of serum TGF $\beta 1 /$ serum CXCL13 was associated with relapses. Multicentre clinical trials and verifications with larger samples might be helpful in order to clarify the relationship between these ratios and prognosis of ANAS. These ratios in this study may indicate a novel way of determining prognosis. In this study, we mainly observed the phenomenon including the difference in factor level in different ANAS groups and between ANAS and normal population. In the future experiment, we will analyse the function and quantity of $\mathrm{T}$ and $\mathrm{B}$ cell subsets by flow cytometry. At the same time, the interaction in different inflammatory factors would be studied to explore the mechanism in depth.

There were many factors associated with the outcomes and relapses of many diseases, and some clinical features have been reported in previous studies. Conscious disturbances and CSF antibody titres have been shown to be predictors of poor outcomes in anti-NMDAR encephaliti s[25], and aggressive immune therapy might reduce the risk of relapses [26]. In our study, the clinical data and cytokines/chemokines in the sera in all ANAS patients were screened as possible predictors during the univariate and multivariate analyses. The factors associated with poor outcomes included having tumours and a chronic onset. No factor was associated with relapses. Meanwhile, we selected all 51 ANAS cases with CSF data and analysed the clinical data and cytokines/chemokines in the sera and CSF. Having tumours was also associated with poor outcomes. The factors associated with relapses included CSF BAFF and the ratio of serum TGF $\beta 1 /$ serum CXCL13. These factors might be linked to the prognosis of ANAS. Thus, to evaluate prognosis of the disease more comprehensively and objectively, we analysed the associated factors including clinical features and cytokines/chemokines.

Much evidence has been collected in previous studies of $\mathrm{AE}$ and PNS [14, 17], and the pathogenesis of neuronal autoantigens has been explored [4]. With the development of neuroimmunology, more antibodies to neuronal autoantigens might be discovered, which may lead to a deeper understanding into the immune mechanisms involved in AE and PNS. In this study, we proposed the concept of "anti-neuronal antibody syndrome" to clarify the pathogenetic relationships. The clinical and immunological aspects of ANAS have been clarified, which might contribute to neuroscientists conducting more profound studies regarding clinical syndromes. Here, we report the data of clinical features and cytokines/chemokines in the sera and CSF, and the data regarding the antibody titres and clinical treatments will be discussed in future studies. Our study focused on the disease on a class of antibodies that target common neuronal epitopes, which was distinguished from most current researches emphasizing one specific antibody. This might promote the further study of ANAS and provide a new idea on clinical study in neuroimmunology.

Our study had the limitations of not being randomized, having fewer cases and having a lack of complete data regarding the cytokines/chemokines in the CSF, which reduces the level of evidence in this ANAS study. Some other factors might influence the levels of cytokines/chemokines found in the sera and CSF, including sampling time, storage conditions, test method, etc. The interactions with different cytokines/chemokines are complex pathological processes. In particular, there is a lack of data on the function and quantity of $\mathrm{T}$ and $\mathrm{B}$ cells. It is difficult to reflect the complex changes in the process of disease solely using the levels or ratios of cytokines/chemokines in the sera or CSF. However, we proposed the clinical range of ANAS, summarized the 
features and cytokines/chemokines in the sera and CSF, and then screened possible predictors associated with outcomes and relapses. This might be helpful for the indepth study of ANAS.

Our study focused on studying the association of disorders with a class of antibodies targeting common neuronal epitopes, and this study highlights the fact that ANAS is a clinical neurological syndrome, not a clinical diagnosis. The term ANAS was proposed for the first time and the clinical range was clarified. The study of ANAS might be a beginning for the understanding of these conditions and should be further promoted. This might provide a novel idea for clinical study in neuroimmunology.

\section{Conclusion}

Our study proposed the term ANAS and clarified the clinical range of this condition. We analysed the association between these diseases, the clinical features and levels of cytokines/chemokines. Then, we screened possible factors associated with poor outcomes, including having tumours, a chronic onset and factors associated with relapses, which included having lower levels of CSF BAFF and a larger ratio of serum TGF $\beta 1 /$ serum CXCL13. These factors might be related to the prognoses of the diseases.

\section{Abbreviations}

ANAS: Anti-neuron antibody syndrome; AE: Autoimmune encephalitis; PNS: Paraneoplastic neurological syndrome; NMDAR: N-methyl-D-aspartate receptor; BAFF: B cell-activating factor; CSF: Cerebrospinal fluid; CXCL: C-X-C motif chemokine; TGF $\beta 1$ : Transforming growth factor $\beta 1$;

EEG: Electroencephalogram; EMG: Electromyogram; mRS: Modified Rankin scale; MRI: Magnetic resonance imaging; FLAIR: Fluid-attenuated inversion recovery; GAD65: 65 kDa glutamic acid decarboxylase; GABAR: $\gamma$-aminobutyric acid receptor

\section{Acknowledgements}

We particularly extend our appreciation to the patients who have been active participants in this clinical study and permitted their samples of sera and CSF to be used.

\section{Authors' contributions}

$\mathrm{BC}, \mathrm{HH}$ and $\mathrm{SL}$ contributed to the study design and literature search. SL and $\mathrm{CL}$ contributed equally to the patient recruitment, data collection, and writing. $\mathrm{XB}$ and $\mathrm{XL}$ contributed to the data analysis and data interpretation. $H G, Y Q$ and SM contributed to the sample collection, experimental implementation, and data interpretation. All authors critically reviewed the manuscript and approved the final draft.

\section{Funding}

This study was supported by the National Natural Science Foundation of China (81671631).

\section{Availability of data and materials}

The datasets generated and analysed during the current study are available from the authors on reasonable request and provided a Data Sharing Agreement approved by the Danish Data Protection Agency.

\section{Declarations}

\section{Ethics approval and consent to participate}

The study was approved by the ethics committee of the 960 Hospital (201611). Written informed consent was obtained from each patient.

\section{Consent for publication}

Not applicable.

\section{Competing interests}

The authors declare that they have no competing interests. No disclosure relevant to the manuscript.

\section{Author details}

${ }^{1}$ Department of Neurology, Shanghai Changhai Hospital, Second Military Medical University, Shanghai, China. ${ }^{2}$ College of Physical Education, Shandong Normal University, Jinan, China. ${ }^{3}$ Department of Neurology, 960 Hospital of the PLA Joint Logistics Support Force, Jinan, China. ${ }^{4}$ Department of Neurology, Chinese PLA General Hospital, Beijing, China.

Received: 12 March 2021 Accepted: 30 August 2021

Published online: 06 December 2021

\section{References}

1. Hara M, Arino H, Petit-Pedrol M, Sabater L, Titulaer MJ, Martinez-Hernandez E, et al. DPPX antibody-associated encephalitis: main syndrome and antibody effects. Neurology. 2017;88:1340-8.

2. Dale RC, Merheb V, Pillai S, Wang D, Cantrill L, Murphy TK, et al. Antibodies to surface dopamine-2 receptor in autoimmune movement and psychiatric disorders. Brain. 2012;135:3453-68.

3. Gaig C, Graus F, Compta Y, Hogl B, Bataller L, Bruggemann N, et al. Clinical manifestations of the anti-IgLON5 disease. Neurology. 2017;88:1736-43.

4. Lancaster E, Dalmau J. Neuronal autoantigens--pathogenesis, associated disorders and antibody testing. Nat Rev Neurol. 2012:8:380-90.

5. Dalmau J, Tuzun E, Wu HY, Masjuan J, Rossi JE, Voloschin A, et al. Paraneoplastic anti-N-methyl-D-aspartate receptor encephalitis associated with ovarian teratoma. Ann Neurol. 2007;61:25-36.

6. Lancaster E, Lai M, Peng X, Hughes E, Constantinescu R, Raizer J, et al. Antibodies to the $G A B A(B)$ receptor in limbic encephalitis with seizures: case series and characterisation of the antigen. Lancet Neurol. 2010;9:67-76.

7. Sanjabi S, Oh SA, Li MO. Regulation of the Immune Response by TGF-beta: From Conception to Autoimmunity and Infection. Cold Spring Harb Perspect Biol. 2017;9(6):a022236.

8. Ouyang W, O'Garra A. IL-10 family cytokines IL-10 and IL-22: from basic science to clinical translation. Immunity. 2019;50:871-91.

9. Kowarik MC, Cepok S, Sellner J, Grummel V, Weber MS, Korn T, et al. CXCL13 is the major determinant for $B$ cell recruitment to the CSF during neuroinflammation. J Neuroinflammation. 2012;9:93.

10. Roberts WK, Blachère NE, Frank MO, Dousmanis A, Ransohoff RM, Darnell RB. A destructive feedback loop mediated by CXCL 10 in central nervous system inflammatory disease. Ann Neurol. 2015;78:619-29.

11. Ferraccioli $\mathrm{G}$, Gremese E. B cell activating factor (BAFF) and BAFF receptors: fakes and facts. Clin Exp Immunol. 2017;190:291-2.

12. Kothur K, Wienholt L, Mohammad SS, Tantsis EM, Pillai S, Britton PN, et al. Utility of CSF Cytokine/Chemokines as Markers of Active Intrathecal Inflammation: Comparison of Demyelinating, Anti-NMDAR and Enteroviral Encephalitis. PLoS One. 2016;11:e0161656.

13. Ding YW, Pan SY, Xie W, Shen HY, Wang HH. Elevated Soluble Fas and FasL in Cerebrospinal Fluid and Serum of Patients With Anti-N-methyl-Daspartate Receptor Encephalitis. Front Neurol. 2018;9:904.

14. Iorio R, Spagni G, Masi G. Paraneoplastic neurological syndromes. Sem Diagn Pathol. 2019;36:279-92.

15. Gresa-Arribas N, Planaguma J, Petit-Pedrol M, Kawachi I, Katada S, Glaser CA et al. Human neurexin-3alpha antibodies associate with encephalitis and alter synapse development. Neurology. 2016;86:2235-42.

16. Graus F, Titulaer MJ, Balu R, Benseler S, Bien CG, Cellucci T, et al. A clinical approach to diagnosis of autoimmune encephalitis. Lancet Neurol. 2016;15: 391-404.

17. Goodfellow JA, Mackay GA. Autoimmune encephalitis. J R Coll Physicians Edinb. 2019:49:287-94. 
18. Titulaer MJ, McCracken L, Gabilondo I, Armangué T, Glaser C, lizuka T, et al. Treatment and prognostic factors for long-term outcome in patients with anti-NMDA receptor encephalitis: an observational cohort study. Lancet Neurol. 2013;12:157-65.

19. Oliveira V, Videira G, Samoes R, Carneiro P, Neves E, Santos E. Paraneoplastic neurological syndromes with onconeural antibodies: A single center retrospective study. J Neurol Sci. 2020;418:117103.

20. Leypoldt F, Hoftberger R, Titulaer MJ, Armangue T, Gresa-Arribas N, Jahn H, et al. Investigations on CXCL13 in anti-N-methyl-D-aspartate receptor encephalitis: a potential biomarker of treatment response. JAMA Neurol. 2015;72:180-6.

21. Khademi M, Kockum I, Andersson ML, lacobaeus E, Brundin L, Sellebjerg F, et al. Cerebrospinal fluid CXCL13 in multiple sclerosis: a suggestive prognostic marker for the disease course. Mult Scler. 2011;17:335-43.

22. Sorensen TL, Trebst C, Kivisakk P. Klaege KL, Majmudar A, Ravid R, et al. Multiple sclerosis: a study of CXCL10 and CXCR3 co-localization in the inflamed central nervous system. J Neuroimmunol. 2002;127:59-68.

23. Pranzatelli MR. Elevated cerebrospinal fluid C-X-C motif chemokine 10 Commonalities in paraneoplastic neurological disorders. Ann Neurol. 2016; 80:792-3.

24. Kothur K, Wienholt L, Brilot F, Dale RC. CSF cytokines/chemokines as biomarkers in neuroinflammatory CNS disorders: A systematic review. Cytokine. 2016;77:227-37.

25. Wang Y, Miao A, Shi Y, Ge J, Wang L, Yu C, et al. Influencing electroclinical features and prognostic factors in patients with anti-NMDAR encephalitis: a cohort follow-up study in Chinese patients. Scientific Rep. 2020;10(1):10753.

26. Nosadini M, Granata T, Matricardi S, Freri E, Ragona F, Papetti L, et al. Italian Working Group on Paediatric Anti Nm-DaRE: Relapse risk factors in anti-Nmethyl-D-aspartate receptor encephalitis. Dev Med Child Neurol. 2019;61: $1101-7$.

\section{Publisher's Note}

Springer Nature remains neutral with regard to jurisdictional claims in published maps and institutional affiliations.

Ready to submit your research? Choose BMC and benefit from:

- fast, convenient online submission

- thorough peer review by experienced researchers in your field

- rapid publication on acceptance

- support for research data, including large and complex data types

- gold Open Access which fosters wider collaboration and increased citations

- maximum visibility for your research: over $100 \mathrm{M}$ website views per year

At $\mathrm{BMC}$, research is always in progress.

Learn more biomedcentral.com/submissions 